Supporting Information

\title{
Comparative Membrane $N$-Glycomics of Different Breast Cancer Cell Lines to Understand Breast Cancer Brain Metastasis
}

\author{
Wenjing Peng ${ }^{1 \S}$, Parvin Mirzaei ${ }^{1 \S}$, Rui Zhu ${ }^{1}$, Shiyue Zhou ${ }^{1}$, Yehia Mechref ${ }^{1 *}$ \\ ${ }^{1}$ Department of Chemistry and Biochemistry, Texas Tech University, Lubbock TX
}

$\S$ Authors contribute equally

*Corresponding Author

Department of Chemistry and Biochemistry

Texas Tech University

Lubbock, TX 79409-1061

Email: yehia.mechref@ttu.edu

Tel: 806-742-3059

Fax: 806-742-1289

Keywords: Glycomics, Breast cancer brain metastasis, Membrane enrichment, Differential expression analysis, LC-MS/MS 


\section{Table of Contents}

Supporting Information Table S1. Putative $N$-glycan structures and their relative abundances derived from different breast cancer cell lines. (This table is presented in an attached Excel file.)

Supporting Information Figure S1. Examples of $N$-glycan identification through LC-MS/MS. (A) Identification of neutral structure $\mathrm{HexNAc}_{3} \mathrm{Hex}_{4}$.

Supporting Information Figure S2. Base peak chromatogram of $N$-glycans derived from different cell line membranes.

Supporting Information Figure S3. Comparison of relative abundances of $N$-glycans whose expressions were significantly changed when compared to 231BR.

Supporting Information Figure S4. Upregulated $N$-glycans in 231BR when compared to 231. 9 out of $12 \mathrm{~N}$-glycans are sialylated.

Supporting Information Figure S5. The relative abundance of LacNAc and LacdiNAc structures observed across six cell lines. 


\section{Supporting Information Figure S1}
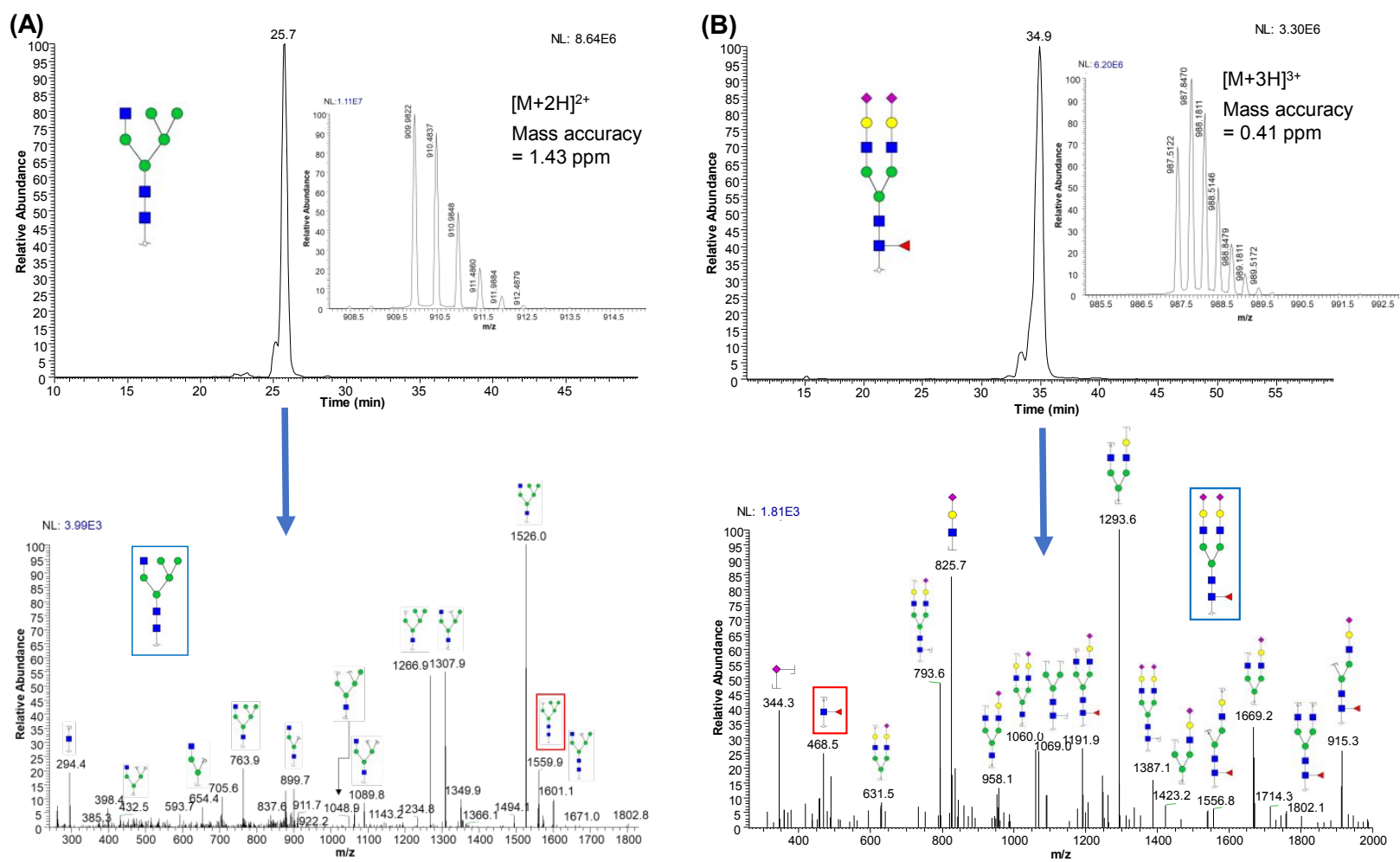

Supporting Information Figure S1. Examples of $N$-glycan identification through LC-MS/MS. (A) Identification of the neutral structure $\mathrm{HexNAc}_{3} \mathrm{Hex}_{4}$. Inset is the full MS. The fragment ion in the red frame is the diagnostic ion indicating there is no galactose attached on the GlcNAc. (B) Identification of the sialylated structure HexNAc $\mathrm{Hex}_{5} \mathrm{DeoxyHex}_{1} \mathrm{NeuAc}_{2}$. Inset is the full MS. The fragment ion in the red frame is the diagnostic ion indicating there is a core-fucosylation. Symbols: $\square, N$-acetylglucosamine (GlcNAc); ○, Galactose (Gal); $\nabla$, Fucose (Fuc); ○, Mannose (Man); $\diamond, N$-acetylneuraminic acid (NeuAc/Sialic Acid). 


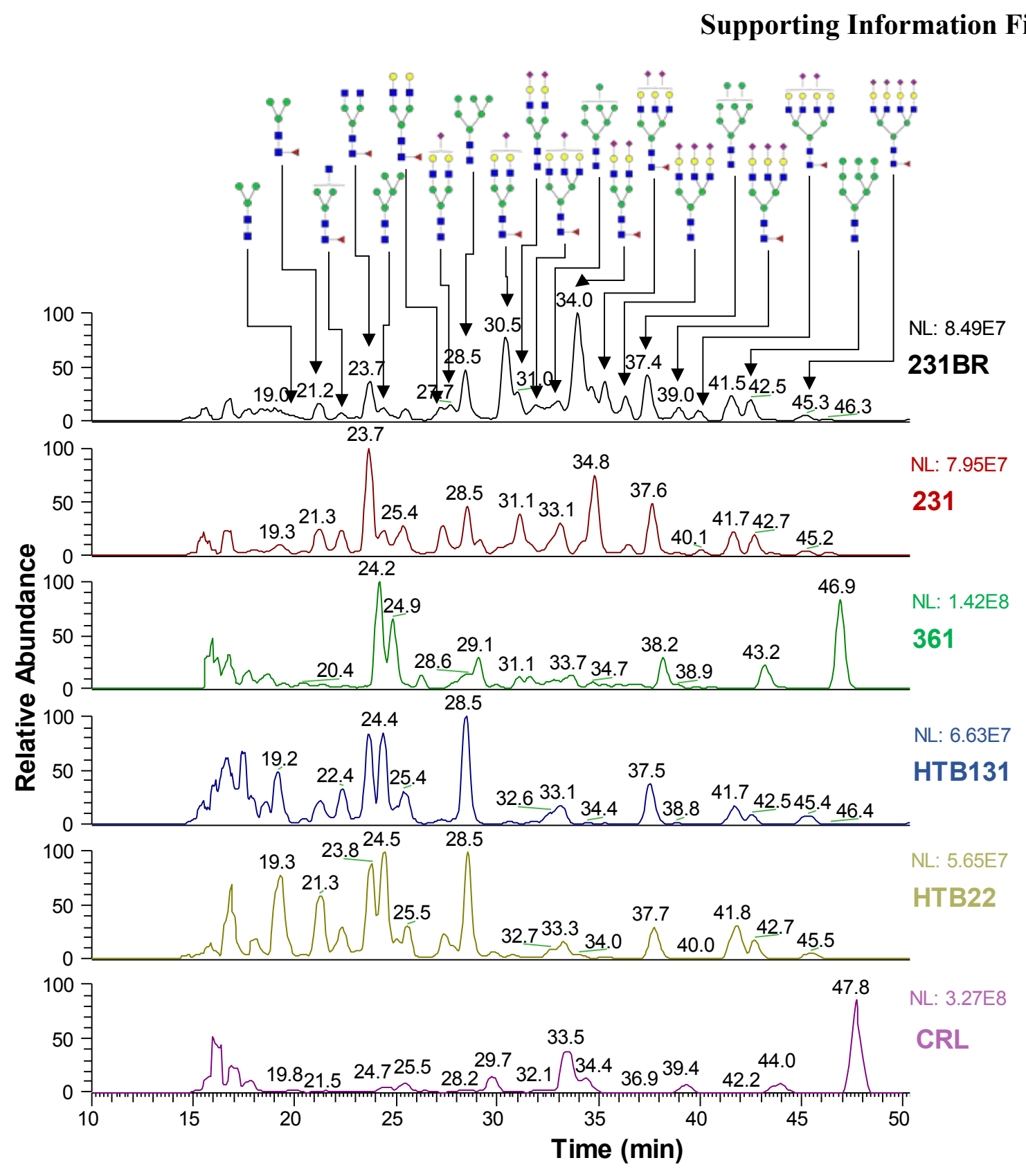

Supporting Information Figure S2. Base peak chromatogram of $N$-glycans derived from different cell line membranes. The major $N$-glycan structure under each peak of the trace of 231BR is assigned. The distinct base peak patterns can be observed across six cell lines. Symbols as in Figure $\mathbf{S 1}$. 


\section{Supporting Information Figure S3}
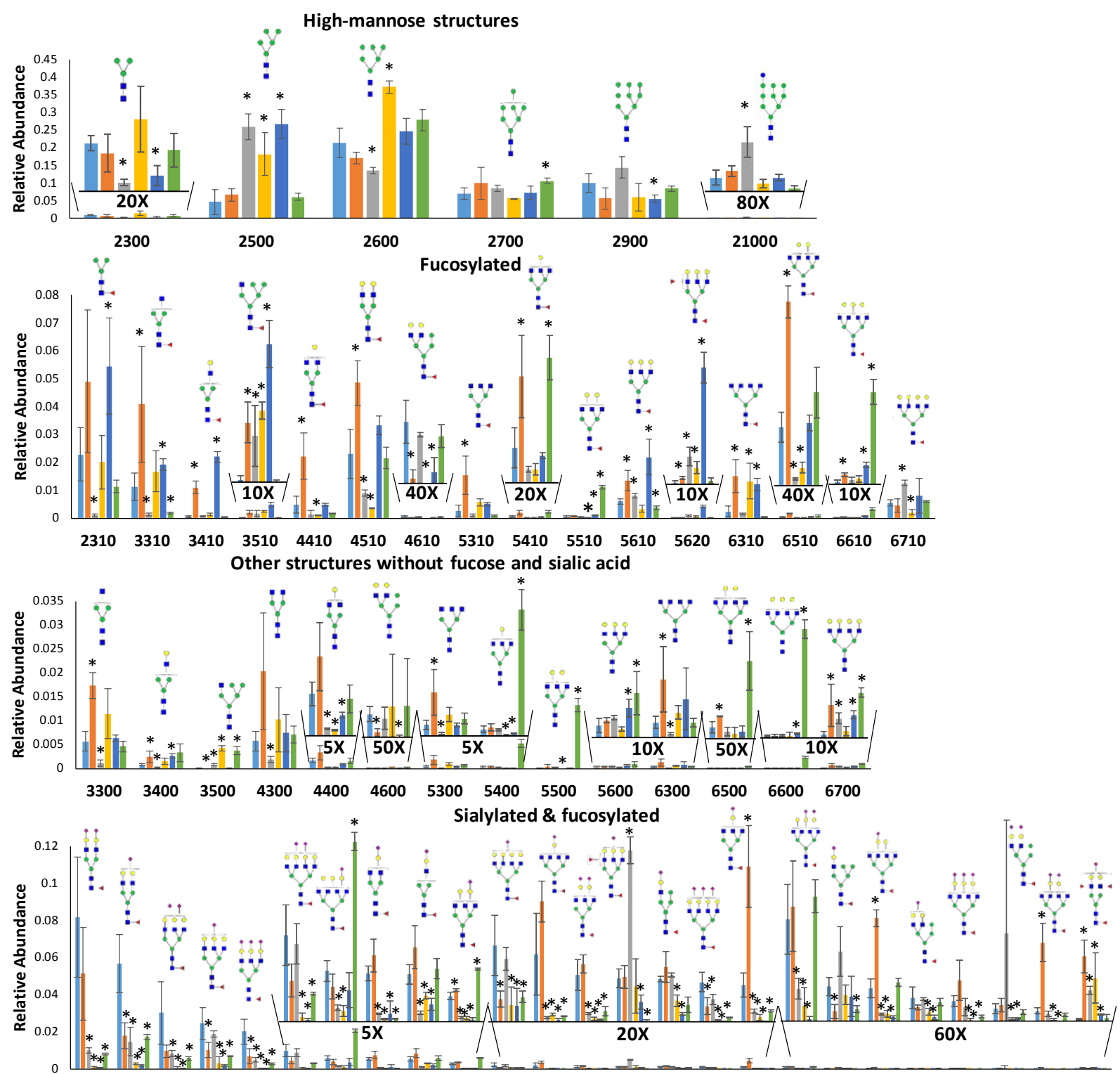

4512451156125611561367126611441134115511671164115512562135116713541166123611651146116613461265124531

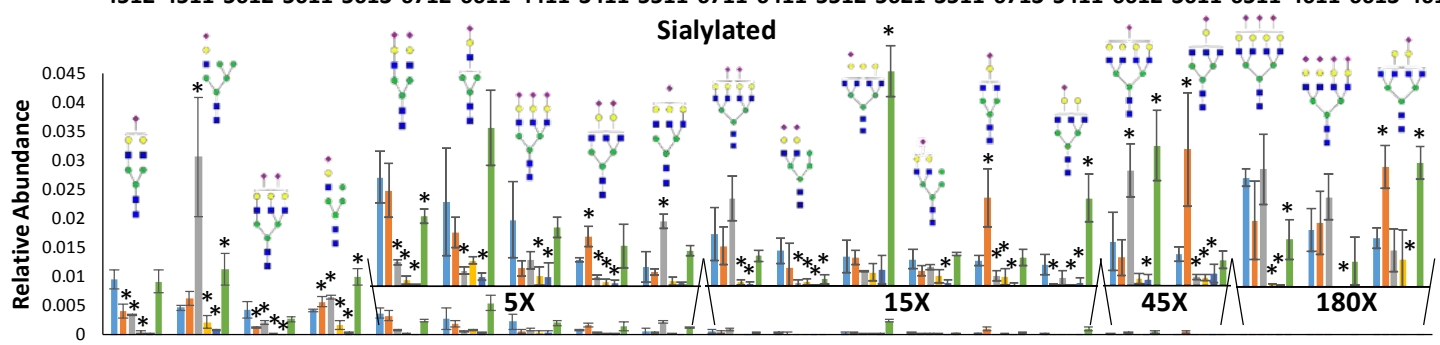

45013601560235014502340156035502560167024602660146014401550167015401670367046501

231BR $\approx 231 \backsim 361 \backsim$ HTB131 $\square$ HTB22 $\square$ CRL

Supporting Information Figure S3. Comparison of relative abundances of $\mathrm{N}$-glycans whose abundances were significantly changed when compared to 231BR. The putative structure was 
assigned to each $N$-glycan composition. * denotes the $N$-glycan relative abundance change is significant when compared to 231BR ( $p<0.05$, based on nonparametric bootstrap test with pooled resampling method, after Bejamini-Hochberg correction). " $X$ " denotes how many times the $\mathrm{Y}$ axis is amplified. For example, "10X" means the $\mathrm{Y}$ axis of that area is amplified 10 times. Nomenclature of $N$-glycans: a 4 digit code is used; the 1 st number denotes the number of Hexosamine (NexNAc); the 2nd number denotes the number of Hexose (Hex); the 3rd number denotes the number of Fucose (DeoxyHex); the 4th number denotes the number of Sialic acid (NeuAc). For instance, 4512 represents $N$-glycan HexNAc${ }_{4} \mathrm{Hex}_{5} \mathrm{DeoxyHex}_{1} \mathrm{NeuAc}_{2}$. Symbols: $\mathrm{N}$-acetylglucosamine (GlcNAc); ○, Galactose (Gal); $\nabla$, Fucose (Fuc); ○, Mannose (Man);,$N$ acetylneuraminic acid (NeuAc/Sialic Acid); $\bullet$, Glucose (Glc). 


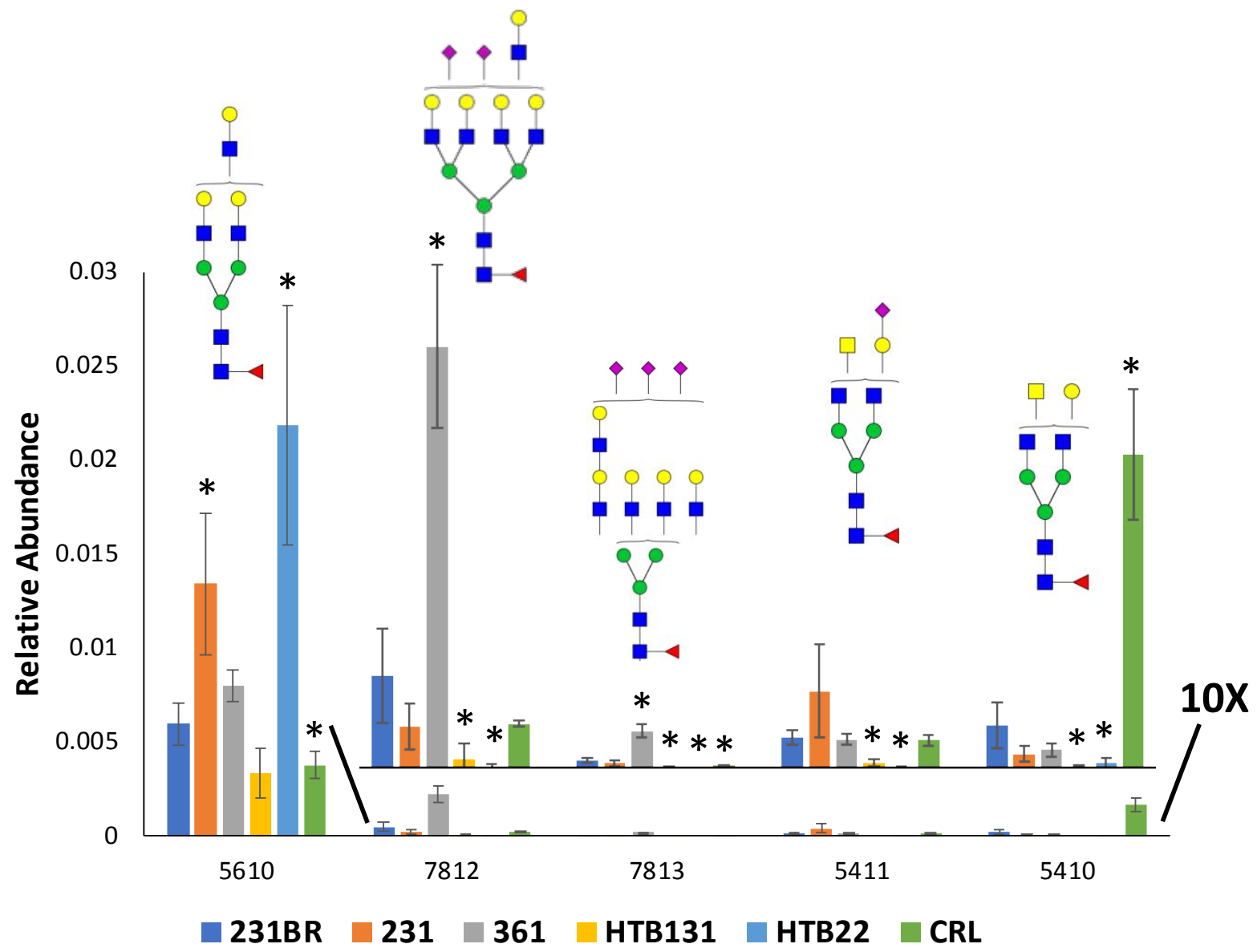

Supporting Information Figure S4. The relative abundance of LacNAc and LacdiNAc structures observed across six cell lines. ${ }^{*}$ denotes that the relative abundance of corresponding structure has a significant difference $(p<0.05)$ when compared to 231BR. "10X" denotes that the $\mathrm{Y}$ axis of corresponding area is amplified 10 times. Nomenclature as in Figure S3. Symbols as in Figure S1. 
Supporting Information Figure S5

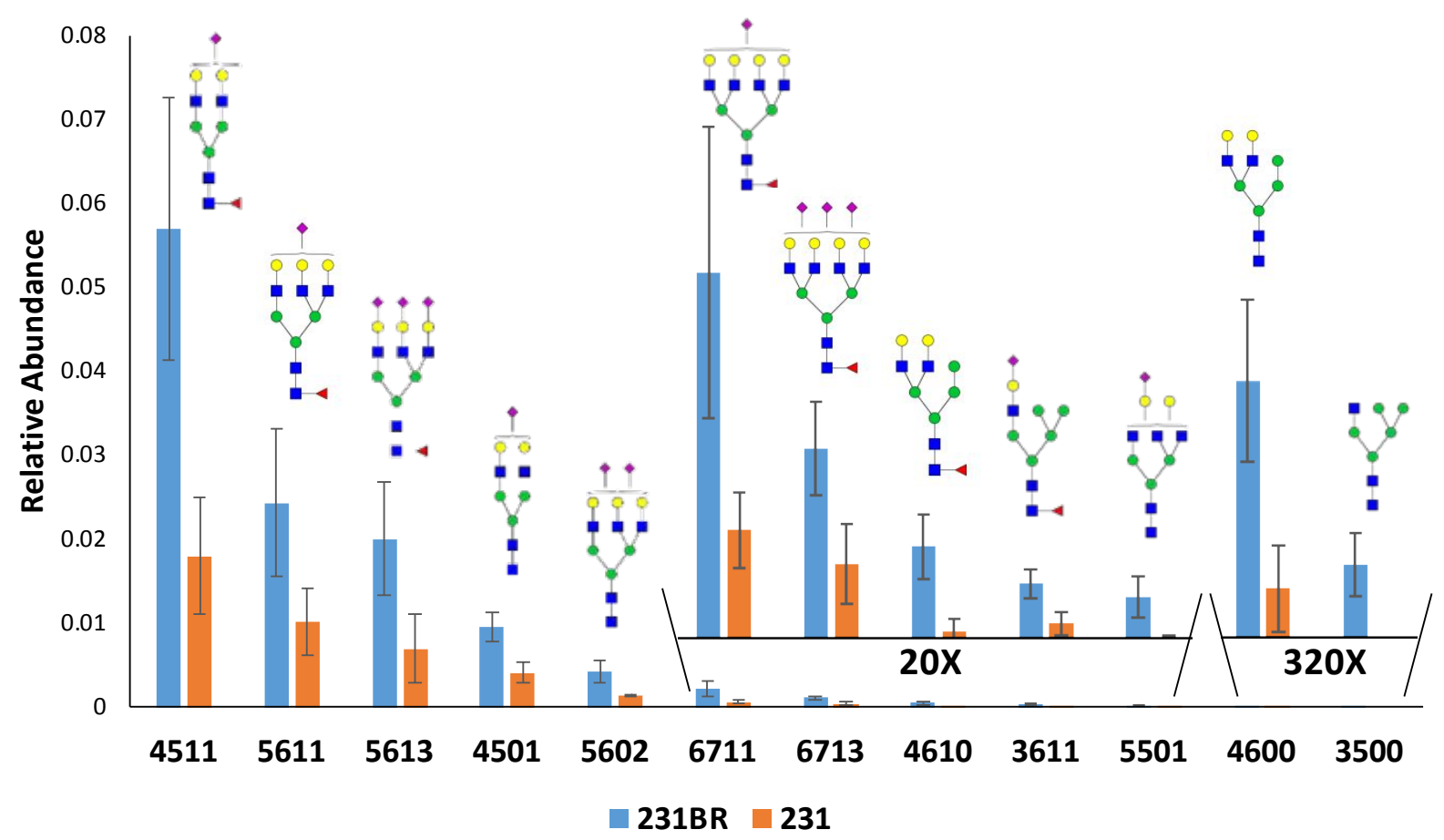

Supporting Information Figure S5. Upregulated $N$-glycans in 231BR when compared to 231. 9 out of $12 \mathrm{~N}$-glycans are sialylated. "X" denotes how many times the Y axis is amplified. For example, "20X" means that the $\mathrm{Y}$ axis of corresponding area is amplified 20 times.

Nomenclature as in Figure S3. Symbols as in Figure S1. 